PROCEEDINGS OF THE

AMERICAN MATHEMATICAL SOCIETY

Volume 128, Number 7, Pages 2171-2179

S 0002-9939(99)05337-X

Article electronically published on November 23, 1999

\title{
A SPECIAL CLASS OF NILMANIFOLDS ADMITTING AN ANOSOV DIFFEOMORPHISM
}

\author{
KAREL DEKIMPE AND WIM MALFAIT
}

(Communicated by Józef Dodziuk)

\begin{abstract}
A nilmanifold admits an Anosov diffeomorphism if and only if its fundamental group (which is finitely generated, torsion-free and nilpotent) supports an automorphism having no eigenvalues of absolute value one. Here we concentrate on nilpotency class 2 and fundamental groups whose commutator subgroup is of maximal torsion-free rank. We prove that the corresponding nilmanifold admits an Anosov diffeomorphism if and only if the torsion-free rank of the abelianization of its fundamental group is greater than or equal to 3 .
\end{abstract}

\section{Introduction}

Let $L$ be a $n$-dimensional connected, simply connected, $c$-step nilpotent Lie group and $\mathfrak{l}$ indicates the (c-step nilpotent, $n$-dimensional) Lie algebra of $L$ over $\mathbb{R}$. Then $\operatorname{Aut}(L)$ is isomorphic to $\operatorname{Aut}(\mathfrak{l})$ via the differential map. If we fix a basis of $\mathfrak{l}$ (as $n$ dimensional vector space), we obtain a faithful representation $\operatorname{Aut}(L) \rightarrow \operatorname{Gl}(n, \mathbb{R})$. An automorphism of $L$ is said to be hyperbolic if and only if its differential has no eigenvalues of absolute value one. Assume $N$ is a lattice of $L$, i.e. a discrete subgroup of $L$ such that $L / N$ is compact. In this case, $N$ is a finitely generated, torsion-free, $c$-step nilpotent group of Hirsch length $n$ and $L$ is the Mal'cev completion of $N$. We refer to $M=L / N$ as the nilmanifold with translation subgroup $N$.

A diffeomorphism of a compact differentiable manifold $M$ without boundary is an Anosov diffeomorphism if and only if, at any point of $M$, the tangent space decomposes as a direct sum of a contracting and an expanding part (for the exact definition, we refer to 1], 5]). It is still an important open problem in dynamical systems (raised by S. Smale in [5]) to classify all smooth compact manifolds admitting Anosov diffeomorphisms. At this moment, the only known manifolds (up to homeomorphism) carrying Anosov diffeomorphisms are algebraic ones, namely tori and flat manifolds, nilmanifolds and infra-nilmanifolds.

In particular, this highlights the problem of classifying all nilmanifolds supporting an Anosov diffeomorphism. On a nilmanifold $M=L / N$, Anosov diffeomorphisms are known to be topologically conjugated to hyperbolic automorphisms of

Received by the editors August 5, 1998.

1991 Mathematics Subject Classification. Primary 58F15, 57R50, 20F34, 20F18.

Key words and phrases. Anosov diffeomorphism, nilmanifold, free 2-step nilpotent Lie algebra, hyperbolic automorphism.

Both authors are Postdoctoral Fellows of the Fund for Scientific Research - Flanders (Belgium) (F.W.O.). 
$L$ which restrict to an automorphism of $N$ ([3]). Hence, deciding that a nilmanifold supports an Anosov diffeomorphism is equivalent to finding a hyperbolic automorphism of its universal covering which restricts to an automorphism of its translation subgroup.

In this paper we concentrate on nilmanifolds whose fundamental group is a 2-step nilpotent group whose commutator subgroup is of maximal torsion-free rank. Such groups are referred to as $T\left(n, \frac{n(n-1)}{2}\right)$-groups. Here $n$ denotes the torsion-free rank of its abelianization (and $\frac{1}{2} n(n-1)$ is the torsion-free rank of its commutator subgroup). In [2], we proved that each nilmanifold with a $T(3,3)$-group as fundamental group admits an Anosov diffeomorphism. Here we generalize this observation and prove that a nilmanifold with a $T\left(n, \frac{n(n-1)}{2}\right)$-group as fundamental group supports an Anosov diffeomorphism if and only if $n \geq 3$.

\section{Preliminaries}

1.1. Notational remarks. If $x, y$ are elements of a group $G$, we make the following convention: $[x, y]=x^{-1} y^{-1} x y$. The lower central series of $G$ is defined inductively by $\gamma_{1}(G)=G$ and $\gamma_{n+1}(G)=\left[\gamma_{n}(G), G\right]\left(n \in \mathbb{N}_{0}\right)$. $G$ is said to be $c$-step nilpotent (or nilpotent of class $c$ ) if and only if $\gamma_{c}(G) \neq\{1\}$ and $\gamma_{c+1}(G)=\{1\}$. The isolator of a subgroup $H$ of $G$ is defined by $\sqrt[G]{H}=\left\{g \in G \mid g^{k} \in H\right.$ for some $\left.k \in \mathbb{N}_{0}\right\}$. It is well known that $\sqrt[G]{\gamma_{k}(G)}$ is characteristic in $G$ and $G / \sqrt[G]{\gamma_{k}(G)}$ is a torsion-free nilpotent group. The lower central series of a Lie algebra $\mathfrak{l}$ is defined analogously as for groups and $\mathfrak{l}$ is $c$-step nilpotent if and only if $\gamma_{c}(\mathfrak{l}) \neq\{1\}$ and $\gamma_{c+1}(\mathfrak{l})=\{1\}$.

1.2. $\boldsymbol{T}(\boldsymbol{n}, \boldsymbol{m})$. If $N$ is a finitely generated, torsion-free, 2-step nilpotent group, then $\sqrt[N]{\gamma_{2}(N)} \cong \mathbb{Z}^{m}$ and $N / \sqrt[N]{\gamma_{2}(N)} \cong \mathbb{Z}^{n}$ for some $m, n \in \mathbb{N}_{0}$. The class of all such groups $N$ is denoted by $T(n, m)$. Obviously $m \leq \frac{1}{2} n(n-1)$. In this paper we study those $N$ whose commutator subgroup is of maximal torsion-free rank; the $T\left(n, \frac{n(n-1)}{2}\right)$-groups.

To each finitely generated, torsion-free, nilpotent group $N$, one can associate its radicable hull $N^{\mathbb{Q}}$ and its Mal'cev completion $N^{\mathbb{R}}$. In fact, $N^{\mathbb{Q}}$ is the torsion-free, divisible nilpotent group containing $N$ as a subgroup and such that every element of $N^{\mathbb{Q}}$ has some positive power lying in $N$, while $N^{\mathbb{R}}$ is the connected, simply connected, nilpotent Lie group containing $N$ as a lattice (i.e. $N$ is a uniform and discrete subgroup of $N^{\mathbb{R}}$ ). Both completions are unique up to isomorphism. Let $\mathfrak{l}^{\mathbb{Q}}$, respectively $\mathfrak{l}^{\mathbb{R}}$, indicate the Lie algebra over $\mathbb{Q}$, respectively over $\mathbb{R}$, of $N^{\mathbb{Q}}$, respectively of $N^{\mathbb{R}}$. We define

Definition 1.1. A Lie group $L$ is of type $T(n, m)$ if and only if $L$ is simply connected, connected, 2-step nilpotent and $\operatorname{dim}\left(L / \gamma_{2}(L)=n\right.$ (i.e. $L / \gamma_{2}(L) \cong \mathbb{R}^{n}$ ) and $\operatorname{dim}\left(\gamma_{2}(L)\right)=m$ (i.e. $\left.\gamma_{2}(L) \cong \mathbb{R}^{m}\right)$.

A Lie algebra $\mathfrak{l}$, over a field $k$, is of type $T(n, m)$ if and only if $\mathfrak{l}$ is 2-step nilpotent and $\operatorname{dim}\left(\mathfrak{l} / \gamma_{2}(\mathfrak{l})=n\right.$ (i.e. $\left.\mathfrak{l} / \gamma_{2}(\mathfrak{l}) \cong k^{n}\right)$ and $\operatorname{dim}\left(\gamma_{2}(\mathfrak{l})\right)=m$ (i.e. $\left.\gamma_{2}(\mathfrak{l}) \cong k^{m}\right)$.

Remark 1.2. Obviously, with the notations introduced above, for a finitely generated, torsion free, nilpotent group $N$, we have that

$$
N \in T(n, m) \Leftrightarrow N^{\mathbb{R}} \text { is of type } T(n, m) \Leftrightarrow \mathfrak{l}^{\mathbb{Q}} \text { and } \mathfrak{l}^{\mathbb{R}} \text { are of type } T(n, m) .
$$




\section{ENDOMORPHISMS OF THE FREE 2-STEP NILPOTENT LIE ALGEBRA}

Recall that the free 2-step nilpotent Lie algebra (over a field $k$ ) on a set $X$ of $n$ generators $X_{1}, X_{2}, \ldots, X_{n}$ is a 2-step nilpotent Lie algebra $\mathfrak{g}$, together with an embedding $i: X=\left\{X_{1}, X_{2}, \ldots, X_{n}\right\} \hookrightarrow \mathfrak{g}$, which is characterised by the following universal property: for any 2-step nilpotent Lie algebra $\mathfrak{h}$ and any map $f: X \rightarrow \mathfrak{h}$, there exists a unique Lie algebra morphism $\varphi_{f}: \mathfrak{g} \rightarrow \mathfrak{h}$ such that $\varphi_{f} \circ i=f$. In the sequel, we omit $i$ and consider the elements $X_{i}$ as belonging to $\mathfrak{g}$. We write $\mathfrak{g}_{n, k}$ for the free 2 -step nilpotent Lie algebra (over a field $k$ ) on $n$ generators.

Lemma 2.1. There is exactly one Lie algebra, over a field $k$, of type $T\left(n, \frac{n(n-1)}{2}\right)$, namely the free 2-step nilpotent Lie algebra $\mathfrak{g}_{n, k}$.

Proof. Let $\mathfrak{l}$ be a Lie algebra of type $T\left(n, \frac{n(n-1)}{2}\right)$. Take a basis $\left\{\bar{B}_{1}, \bar{B}_{2}, \ldots, \bar{B}_{n}\right\}$ of $\mathfrak{l} / \gamma_{2}(\mathfrak{l})$ and choose, for each $i(1 \leq i \leq n)$, an element $B_{i} \in \mathfrak{l}$ which is mapped onto $\bar{B}_{i}$ by the natural projection $\mathfrak{l} \rightarrow \mathfrak{l} / \gamma_{2}(\mathfrak{l})$.

Assume $X_{1}, \ldots, X_{n}$ are the $n$ generators of $\mathfrak{g}_{n, k}$ and define the following map:

$$
f:\left\{X_{1}, X_{2}, \ldots, X_{n}\right\} \rightarrow \mathfrak{l}: X_{i} \mapsto B_{i}, \quad 1 \leq i \leq n .
$$

By the universal property of $\mathfrak{g}_{n, k}$, there exists a (unique) homomorphism $\varphi_{f}$ : $\mathfrak{g}_{n, k} \rightarrow \mathfrak{l}$ such that $\varphi_{f}\left(X_{i}\right)=f\left(X_{i}\right)=B_{i}$, for all $i(1 \leq i \leq n)$. As any of the $B_{i}$ belongs to the image of $\varphi_{f}$, we conclude that $\varphi_{f}$ is surjective. Moreover, as the dimensions of $\mathfrak{g}_{n, k}$ and $\mathfrak{l}$ are equal (both are $\frac{n(n+1)}{2}$ ), $\varphi_{f}$ must be injective too or $\varphi_{f}$ is an isomorphism of Lie algebras.

Corollary 2.2. There is exactly one Lie group, say $L_{n}$, of type $T\left(n, \frac{n(n-1)}{2}\right)$ and any $N \in T\left(n, \frac{n(n-1)}{2}\right)$ is a lattice of $L_{n}$.

There is a natural way of choosing a vector space basis for the Lie algebra $\mathfrak{g}_{n, k}$ : take a set $\left\{X_{1}, X_{2}, \ldots, X_{n}\right\}$ of $n$ generators for $\mathfrak{g}_{n, k}$ and complete this set with all possible commutators of these elements. One obtains the basis $\left\{X_{1}, \ldots, X_{n}, Y_{1}=\right.$ $\left.\left[X_{1}, X_{2}\right], Y_{2}=\left[X_{1}, X_{3}\right], \ldots, Y_{\frac{n(n-1)}{2}}=\left[X_{n-1}, X_{n}\right]\right\}$. In the sequel, we always refer to such a basis as a standard basis of $\mathfrak{g}_{n, k}$ and assume it is ordered as above.

Let us introduce the following:

Definition 2.3. Let $k$ be a field and consider a linear map $f: k^{n} \rightarrow k^{n}$. We say $f$ is induced by an endomorphism $\varphi: \mathfrak{g}_{n, k} \rightarrow \mathfrak{g}_{n, k}$ if and only if there is a basis of $\mathfrak{g}_{n, k} / \gamma_{2}\left(\mathfrak{g}_{n, k}\right)$ (i.e. a good identification of $\mathfrak{g}_{n, k} / \gamma_{2}\left(\mathfrak{g}_{n, k}\right)$ with $\left.k^{n}\right)$ such that

$$
f: \mathfrak{g}_{n, k} / \gamma_{2}\left(\mathfrak{g}_{n, k}\right)\left(\cong k^{n}\right) \rightarrow \mathfrak{g}_{n, k} / \gamma_{2}\left(\mathfrak{g}_{n, k}\right): X+\gamma_{2}\left(\mathfrak{g}_{n, k}\right) \mapsto \varphi(X)+\gamma_{2}\left(\mathfrak{g}_{n, k}\right) .
$$

Of course, any endomorphism $\varphi$ of $\mathfrak{g}_{n, k}$ induces a linear transformation $f$ of $k^{n}$. The converse also holds:

Proposition 2.4. Let $k$ be a field and assume $\left\{Z_{1}, Z_{2}, \ldots, Z_{n}\right\}$ is a basis of $k^{n}$. Let $\left\{X_{i}, Y_{j} \mid 1 \leq i \leq n, 1 \leq j \leq \frac{n(n-1)}{2}\right\}$ be a standard basis of $\mathfrak{g}_{n, k}$.

If a (bijective) linear map $f: k^{n} \rightarrow k^{n}$ is represented by $A_{f} \in k^{n \times n}$ (w.r.t. $\left.\left\{Z_{1}, \ldots, Z_{n}\right\}\right)$, then there a unique $B_{f} \in k^{\frac{n(n-1)}{2} \times \frac{n(n-1)}{2}}$ such that, for any $C \in$ $k^{\frac{n(n-1)}{2} \times n}$, the map $\varphi: \mathfrak{g}_{n, k} \rightarrow \mathfrak{g}_{n, k}$ defined by $\left(\begin{array}{cc}A_{f} & 0 \\ C & B_{f}\end{array}\right)$ (w.r.t. to the standard 
basis of $\mathfrak{g}_{n, k}$ ) is a Lie algebra (auto)morphism of $\mathfrak{g}_{n, k}$ inducing $f$. For $B^{\prime} \neq B_{f}$, there are no $C^{\prime}$ such that $\left(\begin{array}{cc}A_{f} & 0 \\ C^{\prime} & B^{\prime}\end{array}\right)$ defines a $\mathfrak{g}_{n, k}$-endomorphism.

Proof. Assume $f: k^{n} \rightarrow k^{n}$ is a linear map represented by $A_{f}=\left(a_{i, j}\right)$ w.r.t. $\left\{Z_{1}, \ldots, Z_{n}\right\}$. Take $C=\left(c_{i, j}\right) \in k^{\frac{n(n-1)}{2} \times n}$ and construct the following map:

$$
g:\left\{X_{1}, X_{2}, \ldots, X_{n}\right\} \rightarrow \mathfrak{g}_{n, k}: X_{i} \mapsto \sum_{p=1}^{n} a_{p, i} X_{p}+\sum_{q=1}^{\frac{n(n-1)}{2}} c_{q, i} Y_{q} .
$$

Because of the universal property, there exists a unique endomorphism $\varphi: \mathfrak{g}_{n, k} \rightarrow$ $\mathfrak{g}_{n, k}$ (depending on $C$ !) such that, for all $i(1 \leq i \leq n), \varphi\left(X_{i}\right)=g\left(X_{i}\right)$. In other words, for any $C$, there is a unique $B_{f} \in k^{\frac{n(n-1)}{2} \times \frac{n(n-1)}{2}}$ such that the linear map represented by $\left(\begin{array}{cc}A_{f} & 0 \\ C & B_{f}\end{array}\right)$, w.r.t. the given standard basis, is an endomorphism of $\mathfrak{g}_{n, k}$.

We claim that $B_{f}$ is independent of the choice of $C$. It is sufficient to note that $\varphi\left(\left[X_{i}, X_{j}\right]\right)(1 \leq i<j \leq m)$ only depends on $A$ :

$$
\varphi\left(\left[X_{i}, X_{j}\right]\right)=\left[\varphi\left(X_{i}\right), \varphi\left(X_{j}\right)\right]=\left[\sum_{p=1}^{n} a_{p, i} X_{p}, \sum_{p=1}^{n} a_{p, j} X_{p}\right] .
$$

Obviously, $\varphi$ is surjective (and hence bijective) if and only if $\left\{\varphi\left(X_{1}\right), \ldots, \varphi\left(X_{m}\right)\right\}$ generates $\mathfrak{g}_{n, k}$ and this is equivalent to $f$ being surjective (and so bijective).

The following proposition establishes interesting information on the eigenvalues of endomorphisms of $\mathfrak{g}_{n, k}$.

Proposition 2.5. Let $k$ be a field and fix a standard basis $\left\{X_{i}, Y_{j} \mid 1 \leq i \leq n, 1 \leq\right.$ $\left.j \leq \frac{n(n-1)}{2}\right\}$ of $\mathfrak{g}_{n, k}$. Assume $\varphi$ is an endomorphism of $\mathfrak{g}_{n, k}$ represented by $\left(\begin{array}{cc}A & 0 \\ C & B\end{array}\right)$ with $A \in k^{n \times n}, B \in k^{\frac{n(n-1)}{2} \times \frac{n(n-1)}{2}}$ and $C \in k^{\frac{n(n-1)}{2} \times n}$.

If $\left\{\lambda_{1}, \lambda_{2}, \ldots, \lambda_{n}\right\}$ is the set of eigenvalues of $A$, then $\left\{\lambda_{i} \lambda_{j} \mid 1 \leq i<j \leq n\right\}$ is the set of eigenvalues of $B$ and hence $\operatorname{det}(B)=\operatorname{det}(A)^{n-1}$. Moreover, if $k$ is of characteristic zero and the characteristic polynomial of $A$ has integer coefficients, then the characteristic polynomial of $B$ also has integer coefficients.

Proof. Without loss of generality, we may assume that $k$ is algebraically closed. Then, over $k, A$ can be put in lower triangular form or there exists a standard basis

$$
X_{1}^{\prime}, X_{2}^{\prime}, \ldots, X_{n}^{\prime}, Y_{1}^{\prime}=\left[X_{1}^{\prime}, X_{2}^{\prime}\right], Y_{2}^{\prime}=\left[X_{1}^{\prime}, X_{3}^{\prime}\right], \ldots, Y_{\frac{n(n-1)}{2}}^{\prime}=\left[X_{n-1}^{\prime}, X_{n}^{\prime}\right]
$$

of $\mathfrak{g}_{n, k}$ such that $\varphi$ is represented by $\left(\begin{array}{cc}A^{\prime} & 0 \\ C^{\prime} & B^{\prime}\end{array}\right)$ w.r.t. (11) and

$$
A^{\prime}=\left(\begin{array}{cccc}
\lambda_{1} & 0 & \cdots & 0 \\
* & \lambda_{2} & \cdots & 0 \\
\vdots & \vdots & \ddots & \vdots \\
* & * & \cdots & \lambda_{n}
\end{array}\right)
$$

Fix $Y_{j}^{\prime}=\left[X_{k}^{\prime}, X_{l}^{\prime}\right]\left(1 \leq j \leq \frac{n(n-1)}{2}\right)$ for some $k, l(1 \leq k<l \leq n)$. 
Compute that

$$
\begin{aligned}
\varphi\left(Y_{j}^{\prime}\right)= & \varphi\left(\left[X_{k}^{\prime}, X_{l}^{\prime}\right]\right)=\left[\varphi\left(X_{k}^{\prime}\right), \varphi\left(X_{l}^{\prime}\right)\right] \\
= & {\left[\lambda_{k} X_{k}^{\prime}+\sum_{r=k+1}^{n} \alpha_{r} X_{r}^{\prime}, \lambda_{l} X_{l}^{\prime}+\sum_{s=l+1}^{n} \beta_{s} X_{s}^{\prime}\right] } \\
& \left(\text { for some } \alpha_{r}, \beta_{s} \in k\right) \\
= & \lambda_{k} \lambda_{l} Y_{j}^{\prime}+\underbrace{\sum_{s=l+1}^{n} \lambda_{k} \beta_{s}\left[X_{k}^{\prime}, X_{s}^{\prime}\right]+\sum_{r=k+1}^{n} \alpha_{r} \lambda_{l}\left[X_{r}^{\prime}, X_{l}^{\prime}\right]+\sum_{r=k+1}^{n} \sum_{s=l+1}^{n} \alpha_{r} \beta_{s}\left[X_{r}^{\prime}, X_{s}^{\prime}\right]}_{\text {linear combination of basis vectors to the right of } Y_{j}^{\prime} \text { in } \mathbb{1}}
\end{aligned}
$$

or

$$
B^{\prime}=\left(\begin{array}{cccc}
\lambda_{1} \lambda_{2} & 0 & \cdots & 0 \\
* & \lambda_{1} \lambda_{3} & \cdots & 0 \\
\vdots & \vdots & \ddots & \vdots \\
* & * & \cdots & \lambda_{n-1} \lambda_{n}
\end{array}\right) .
$$

It follows immediately that the eigenvalues of $B^{\prime}$ (and so the eigenvalues of $B$ ) are

$$
\delta_{1}=\lambda_{1} \lambda_{2}, \delta_{2}=\lambda_{1} \lambda_{3}, \ldots, \delta_{\frac{n(n-1)}{2}}=\lambda_{n-1} \lambda_{n} .
$$

And as the determinant of $B$ is the product of all its eigenvalues,

$$
\operatorname{det}(B)=\delta_{1} \delta_{2} \cdots \delta_{\frac{n(n-1)}{2}}=\lambda_{1}^{n-1} \lambda_{2}^{n-1} \cdots \lambda_{n}^{n-1}=(\operatorname{det}(A))^{n-1} .
$$

Now assume $k$ is of characteristic 0 and suppose that the characteristic polynomial of $A$, say $\mu_{A}(x)$, has integer coefficients. Since

$$
\begin{aligned}
\mu_{A}(x) & =\prod_{i=1}^{n}\left(x-\lambda_{i}\right) \\
& =x^{n}-\left(\sum_{i=1}^{n} \lambda_{i}\right) x^{n-1}+\left(\sum_{i, j=1, i<j}^{n} \lambda_{i} \lambda_{j}\right) x^{n-2}+\cdots+(-1)^{n} \prod_{i=1}^{n} \lambda_{i},
\end{aligned}
$$

all elementary symmetric polynomials $\sigma_{i}\left(x_{1}, \ldots, x_{n}\right)(1 \leq i \leq n)$ in $n$ variables take integer values when we substitute $x_{i}$ by $\lambda_{i}$. The fundamental theorem on symmetric polynomials states that any symmetric polynomial over $\mathbb{Z}$, in $n$ variables, belongs to the polynomial ring $\mathbb{Z}\left[\sigma_{1}, \sigma_{2}, \ldots, \sigma_{n}\right]$ or, for any symmetric polynomial $\sigma\left(x_{1}, \ldots, x_{n}\right)$ over $\mathbb{Z}, \sigma\left(\lambda_{1}, \ldots, \lambda_{n}\right) \in \mathbb{Z}$.

Consider the following polynomial in $n+1$ variables $x, x_{1}, x_{2}, \ldots, x_{n}$ :

$$
\left(x-x_{1} x_{2}\right)\left(x-x_{1} x_{3}\right) \cdots\left(x-x_{n-1} x_{n}\right)=\sum_{i=0}^{\frac{n(n-1)}{2}} c_{i}\left(x_{1}, \ldots, x_{n}\right) x^{i}
$$

where obviously all $c_{i}\left(x_{1}, x_{2}, \ldots, x_{n}\right)\left(0 \leq i \leq \frac{n(n-1)}{2}\right)$ are symmetric polynomials over $\mathbb{Z}$ and hence, $c_{i}\left(\lambda_{1}, \lambda_{2}, \ldots, \lambda_{n}\right) \in \mathbb{Z}$. We conclude that the characteristic polynomial $\mu_{B}(x)=\prod_{i, j=1, i<j}^{n}\left(x-\lambda_{i} \lambda_{j}\right)$ of $B$ has integer coefficients. 


\section{Hyperbolic AUtOMORPHISMS ON $T\left(n, \frac{n(n-1)}{2}\right)$-GROUPS}

Let $N \in T\left(n, \frac{n(n-1)}{2}\right)$. Fix elements $a_{1}, \ldots, a_{n}, b_{1}, \ldots, b_{\frac{n(n-1)}{2}}$ of $N$ such that $b_{1}, \ldots, b_{\frac{n(n-1)}{2}}$ generate $\Gamma=\sqrt[N]{\gamma_{2}(N)} \cong \mathbb{Z}^{\frac{n(n-1)}{2}}$ and $a_{1} \Gamma, \ldots, a_{n} \Gamma$ generate $N / \Gamma \cong$ $\mathbb{Z}^{n}$. We refer to such a set of generators of $N$ as an adapted set of generators.

Identify the Lie algebra (over $\mathbb{Q}$ ) of the radicable hull $N^{\mathbb{Q}}$ of $N$ with the free 2-step nilpotent Lie algebra $\mathfrak{g}_{n, \mathbb{Q}}$ (Lemma 2.1). Under the map $N \hookrightarrow N^{\mathbb{Q}} \stackrel{\log }{\longrightarrow} \mathfrak{g}_{n, \mathbb{Q}}$ the elements $b_{j}\left(1 \leq j \leq \frac{n(n-1)}{2}\right)$ are mapped into $\gamma_{2}\left(\mathfrak{g}_{n, \mathbb{Q}}\right)$. Clearly, if

$$
A_{i}=\log \left(a_{i}\right) \quad(1 \leq i \leq n), \quad B_{j}=\log \left(b_{j}\right) \quad\left(1 \leq j \leq \frac{n(n-1)}{2}\right),
$$

then

1. $A_{1}, A_{2}, \ldots, A_{n}$ freely generate the Lie algebra $\mathfrak{g}_{n, \mathbb{Q}}$,

2. $\left\{B_{1}, B_{2}, \ldots, B_{\underline{n(n-1)}}\right\}$ is a vector space basis of $\gamma_{2}\left(\mathfrak{g}_{n, \mathbb{Q}}\right)$ and

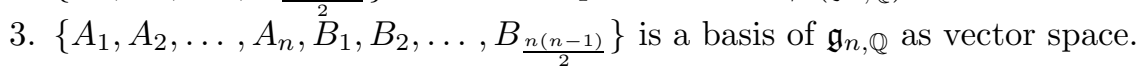

An automorphism $\nu$ of $N$ defines matrices $A_{\nu}=\left(\alpha_{i, j}\right) \in \operatorname{Gl}(n, \mathbb{Z}), B_{\nu}=\left(\beta_{i, j}\right) \in$ $\operatorname{Gl}\left(\frac{n(n-1)}{2}, \mathbb{Z}\right)$ and $C_{\nu}=\left(\gamma_{i, j}\right) \in \mathbb{Z}^{\frac{n(n-1)}{2} \times n}$ such that

$$
\nu\left(a_{i}\right)=\prod_{p=1}^{n} a_{p}^{\alpha_{p, i}} \prod_{q=1}^{\frac{n(n-1)}{2}} b_{q}^{\gamma_{q, i}} \text { and } \quad \nu\left(b_{j}\right)=\prod_{q=1}^{\frac{n(n-1)}{2}} b_{q}^{\beta_{q, j}}
$$

where $1 \leq i \leq n$ and $1 \leq j \leq \frac{n(n-1)}{2}$. We say $\nu \in \operatorname{Aut}(N)$ is determined by the matrices $A_{\nu}, B_{\nu}, C_{\nu}$. Of course, not each triple $A, B, C(A \in \operatorname{Gl}(n, \mathbb{Z}), B \in$ $\left.\mathrm{Gl}\left(\frac{n(n-1)}{2}, \mathbb{Z}\right), C \in \mathbb{Z}^{\frac{n(n-1)}{2} \times n}\right)$ determines an automorphism of $N$.

Each automorphism $\nu$ of $N$ induces unique automorphisms $\tilde{\nu}$ of $N^{\mathbb{Q}}$ and $\phi_{\nu}$ of $\mathfrak{g}_{n, \mathbb{Q}}$ such that the following diagram commutes:

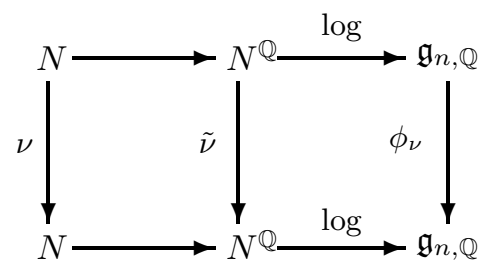

Conversely, for each automorphism $\varphi$ of $\mathfrak{g}_{n, \mathbb{Q}}$, there is a unique $\tilde{\varphi} \in \operatorname{Aut}\left(N^{\mathbb{Q}}\right) \operatorname{such}$ that $\log \circ \tilde{\varphi}=\varphi \circ \log$. Observe that

Lemma 3.1. Let $N \in T\left(n, \frac{n(n-1)}{2}\right)$ and $\nu \in \operatorname{Aut}(N)$. Assume $\nu$ is determined by matrices $A_{\nu}, B_{\nu}, C_{\nu}$ w.r.t. an adapted set $\left\{a_{1}, \ldots, a_{n}, b_{1}, \ldots, b_{\frac{n(n-1)}{2}}\right\}$ of generators of $N$. Then the corresponding automorphism $\phi_{\nu}$ of $\mathfrak{g}_{n, \mathbb{Q}}$ is represented by $\left(\begin{array}{cc}A_{\nu} & 0 \\ D_{\nu} & B_{\nu}\end{array}\right)$, for some $D_{\nu} \in \mathbb{Q}^{\frac{n(n-1)}{2} \times n}$, w.r.t. $\left\{A_{1}=\log \left(a_{1}\right), \ldots, A_{n}=\log \left(a_{n}\right)\right.$, $\left.B_{1}=\log \left(b_{1}\right), \ldots, B_{\frac{n(n-1)}{2}}=\log \left(b_{\frac{n(n-1)}{2}}\right)\right\}$. 
Proof. Because of the Campbell-Baker-Hausdorff formula, for all $i(1 \leq i \leq n)$,

$$
\begin{aligned}
\phi_{\nu}\left(A_{i}\right) & =\log \left(\nu\left(a_{i}\right)\right)=\log \left(\prod_{p=1}^{n} a_{p}^{\alpha_{p, i}} \prod_{q=1}^{\frac{n(n-1)}{2}} b_{q}^{\gamma_{q, i}}\right) \\
& =\sum_{p=1}^{n} \log \left(a_{p}^{\alpha_{p, i}}\right)+D_{i}=\sum_{p=1}^{n} \alpha_{p, i} A_{p}+D_{i},
\end{aligned}
$$

for some element $D_{i} \in \gamma_{2}\left(\mathfrak{g}_{n, \mathbb{Q}}\right)$. And for all $j\left(1 \leq j \leq \frac{n(n-1)}{2}\right)$,

$$
\phi_{\nu}\left(B_{j}\right)=\log \left(\nu\left(b_{j}\right)\right)=\log \left(\prod_{q=1}^{\frac{n(n-1)}{2}} b_{q}^{\beta_{q, j}}\right)=\sum_{q=1}^{\frac{n(n-1)}{2}} \beta_{q, j} B_{q} .
$$

An interesting property (implicitly mentioned in [4]) is the following:

Lemma 3.2. If the characteristic polynomial of $K \in \mathrm{Gl}(n, \mathbb{Q})$ has integer coefficients and unit constant term, then there exists $l \in \mathbb{N}_{0}$ such that $K^{l} \in \operatorname{Gl}(n, \mathbb{Z})$.

This lemma is crucial to prove that

Theorem 3.3. A $T\left(n, \frac{n(n-1)}{2}\right)$-group admits a hyperbolic automorphism if and only if there is a matrix $A \in \operatorname{Gl}(n, \mathbb{Z})$ with eigenvalues $\lambda_{1}, \lambda_{2}, \ldots, \lambda_{n}$ such that, for all $i, j(1 \leq i, j \leq n),\left|\lambda_{i} \lambda_{j}\right| \neq 1$.

Proof. Let $N \in T\left(n, \frac{n(n-1)}{2}\right)$ and assume $\left\{a_{1}, \ldots, a_{n}, b_{1}, \ldots b_{\underline{n(n-1)}}\right\}$ is an adapted set of generators of $N$. Write $A_{i}=\log \left(a_{i}\right), B_{j}=\log \left(b_{j}\right)(1 \leq i \leq n, 1 \leq j \leq$ $\left.\frac{n(n-1)}{2}\right)$. The change of basis between

$$
A_{1}, A_{2}, \ldots, A_{n}, B_{1}, B_{2}, \ldots, B_{\frac{n(n-1)}{2}}
$$

and the standard basis

$$
A_{1}, A_{2}, \ldots, A_{n},\left[A_{1}, A_{2}\right],\left[A_{1}, A_{3}\right], \ldots,\left[A_{n-1}, A_{n}\right]
$$

of $\mathfrak{g}_{n, \mathbb{Q}}$ is given by a matrix of the form $\left(\begin{array}{ll}I & 0 \\ 0 & Q\end{array}\right)$ with $Q \in \operatorname{Gl}\left(\frac{n(n-1)}{2}, \mathbb{Q}\right.$ ) (where $I$ denotes the $n \times n$ identity matrix).

Assume $N$ admits a hyperbolic automorphism $\nu$, determined by matrices $A_{\nu}$, $B_{\nu}$ and $C_{\nu}$ (w.r.t. $\left\{a_{1}, \ldots, a_{n}, b_{1}, \ldots b_{\frac{n(n-1)}{2}}\right\}$ ). Since the corresponding automorphism $\phi_{\nu}$ of $\mathfrak{g}_{n, \mathbb{Q}}$ is represented (w.r.t. (2), Lemma 3.1) by $\left(\begin{array}{cc}A_{\nu} & 0 \\ D_{\nu} & B_{\nu}\end{array}\right)$, for some $D_{\nu} \in \mathbb{Q}^{\frac{n(n-1)}{2} \times n}, A_{\nu}$ and $B_{\nu}$ are both hyperbolic. W.r.t. (31), $\phi_{\nu}$ is represented by $\left(\begin{array}{cc}A_{\nu} & 0 \\ D^{\prime} & Q B_{\nu} Q^{-1}\end{array}\right)$, for some $D^{\prime} \in \mathbb{Q}^{\frac{n(n-1)}{2} \times n}$. Because of Proposition 2.5, the eigenvalues of $Q B_{\nu} Q^{-1}$ (and equivalently of $B_{\nu}$ ) are $\lambda_{i} \lambda_{j}(1 \leq i<j \leq n)$ if $\lambda_{1}, \ldots, \lambda_{n}$ are the eigenvalues of $A_{\nu}$. As $A_{\nu}$ and $B_{\nu}$ are hyperbolic, it follows that, for all $i, j$ $(1 \leq i, j \leq n),\left|\lambda_{i} \lambda_{j}\right| \neq 1$.

Conversely, assume $A \in \operatorname{Gl}(n, \mathbb{Z})$ and its eigenvalues $\lambda_{1}, \ldots, \lambda_{n}$ satisfy $\left|\lambda_{i} \lambda_{j}\right| \neq$ 1 , for all $i, j(1 \leq i, j \leq n)$. Let $B^{\prime}$ be the unique element of $\operatorname{Gl}\left(\frac{n(n-1)}{2}, \mathbb{Q}\right)$ such that, for any $C^{\prime} \in \mathbb{Q}^{\frac{n(n-1)}{2} \times n}$, the linear map represented by $\left(\begin{array}{cc}A & 0 \\ C^{\prime} & B^{\prime}\end{array}\right)$ (w.r.t. 
(3)) ) is an automorphism $\phi$ of $\mathfrak{g}_{n, \mathbb{Q}}$ (Proposition [2.4). W.r.t. (2), $\phi$ is represented by $\left(\begin{array}{ll}A & 0 \\ C & B\end{array}\right)$ for some $C \in \mathbb{Q}^{\frac{n(n-1)}{2} \times n}$ and with $B=Q^{-1} B^{\prime} Q$. We know that the characteristic polynomial of $B^{\prime}$ (and equivalently of $B$ ) has integer coefficients and unit constant term (Proposition 2.5). Hence, there exists $k \in \mathbb{N}$ such that $B^{k} \in$ $\operatorname{Gl}(n, \mathbb{Z})$ (Lemma 3.2). Then $\left(\begin{array}{cc}A^{k} & 0 \\ C^{\prime \prime} & B^{\prime k}\end{array}\right)$, for any $C^{\prime \prime} \in \mathbb{Q}^{\frac{n(n-1)}{2} \times n}$, represents, w.r.t. (3), an automorphism $\varphi$ of $\mathfrak{g}_{n, \mathbb{Q}}$ (Proposition 2.4) and the diagonal blocks of $\varphi$, represented w.r.t. (2), are always $A^{k}$ and $B^{k}$ (both have integral entries). Hence, the eigenvalues of $\varphi$ are $\lambda_{1}^{k}, \ldots, \lambda_{n}^{k}, \lambda_{1}^{k} \lambda_{2}^{k}, \ldots, \lambda_{n-1}^{k} \lambda_{n}^{k}$ (Proposition 2.5) and none of them is of absolute value one. Write $\tilde{\varphi}$ for the corresponding automorphism of $N^{\mathbb{Q}}$. Since $B^{k}$ is a matrix over $\mathbb{Z}$, the images $\tilde{\varphi}\left(b_{j}\right)$ can be seen as elements of $N$ $\left(1 \leq j \leq \frac{n(n-1)}{2}\right)$. Moreover, for a suitable $C^{\prime \prime}$, also $\tilde{\varphi}\left(a_{i}\right) \in N(1 \leq i \leq n)$ and we obtain a hyperbolic automorphism $\tilde{\varphi}$ of $N$.

Remark 3.4. The reader might have the feeling that the results obtained so far generalize towards groups of higher nilpotency class but there are some subtle obstacles to establish this. However, as the first author hopes to show in a forthcoming paper, it is possible, using a different approach, to build up a somehow weaker version of the preceding results for nilpotency classes greater than 2 .

Inspired by Theorem 3.3 , we look for matrices over $\mathbb{Z}$ satisfying the equivalent algebraic condition formulated above.

Proposition 3.5. There exists a matrix $A \in \operatorname{Gl}(n, \mathbb{Z})$ with eigenvalues $\lambda_{1}, \ldots, \lambda_{n}$ such that $\left|\lambda_{i} \lambda_{j}\right| \neq 1$ for all $i, j(1 \leq i, j \leq n)$ if and only if $n \geq 3$.

Proof. Obviously, if $n \leq 2$, then there are no suitable $A \in \operatorname{Gl}(n, \mathbb{Z})$. For $n=3$,

$$
A_{0}=\left(\begin{array}{lll}
1 & 1 & 1 \\
1 & 2 & 2 \\
1 & 2 & 3
\end{array}\right)
$$

satisfies the desired condition since its eigenvalues (we always round to 6 decimal places) are $\lambda_{1}=5.048917, \lambda_{2}=0.307978$ and $\lambda_{3}=0.643104$. For $n=4$, one can use

$$
A_{1}=\left(\begin{array}{cccc}
0 & 1 & 0 & 0 \\
0 & 0 & 1 & 0 \\
0 & 0 & 0 & 1 \\
-1 & 6 & -1 & -5
\end{array}\right)
$$

with eigenvalues $\lambda_{4}=0.176619, \lambda_{5}=0.824821, \lambda_{6}=-4.463566, \lambda_{7}=-1.537874$. For $n=5$,

$$
A_{2}=\left(\begin{array}{lllll}
1 & 1 & 1 & 1 & 1 \\
1 & 2 & 2 & 2 & 2 \\
1 & 2 & 3 & 3 & 3 \\
1 & 2 & 3 & 4 & 4 \\
1 & 2 & 3 & 4 & 5
\end{array}\right)
$$

with eigenvalues $\lambda_{8}=0.271554, \lambda_{9}=0.353253, \lambda_{10}=0.582964, \lambda_{11}=1.44869$ and $\lambda_{12}=12.3435$, is suitable. Moreover, one easily checks that $\lambda_{1}, \lambda_{2}, \ldots, \lambda_{12}$ satisfy $\left|\lambda_{i} \lambda_{j}\right| \neq 1$, for all $i, j(1 \leq i, j \leq 12)$. 
If $n>5$, say $n=3 k+l$ with $k \geq 2$ and $l \in\{0,1,2\}$, consider

$$
A=\left(\begin{array}{ccccc}
A_{0} & 0 & \cdots & 0 & 0 \\
0 & A_{0} & \cdots & 0 & 0 \\
\vdots & \vdots & \ddots & \vdots & \vdots \\
0 & 0 & \cdots & A_{0} & 0 \\
0 & 0 & \cdots & 0 & A_{l}
\end{array}\right) \in \operatorname{Gl}(n, \mathbb{Z})
$$

where the diagonal consists of $k-1$ copies of $A_{0}$ followed by one copy of $A_{l}$. Since the eigenvalues of $A$ all belong to $\left\{\lambda_{1}, \lambda_{2}, \ldots, \lambda_{12}\right\}$, this finishes our proof.

We can conclude that

Theorem 3.6. A $T\left(n, \frac{n(n-1)}{2}\right)$-group admits a hyperbolic automorphism if and only if $n \geq 3$.

Or equivalently, a nilmanifold whose fundamental group is a $T\left(n, \frac{n(n-1)}{2}\right)$-group admits an Anosov diffeomorphism if and only if $n \geq 3$.

For explicit examples illustrating our results, we refer to [2].

\section{REFERENCES}

[1] Anosov, D. Geodesic flow on closed Riemannian manifolds with negative curvature. Proc. Steklov Inst., 90, 1969. MR 36:7157

[2] Malfait, W. Anosov diffeomorphisms on nilmanifolds of dimension at most six. 1998. Preprint.

[3] Manning, A. There are no new Anosov diffeomorphisms on tori. Amer. J. Math., 1974, 96 (3), pp. 422-429. MR 50:11324]

[4] Porteous, H. L. Anosov diffeomorphisms of flat manifolds. Topology, 1972, 11, pp. 307-315. MR 45:6035

[5] Smale, S. Differentiable dynamical systems. Bull. Amer. Math. Soc., 1967, 73, pp. 747-817. MR 37:3598

Department of Mathematics, Katholieke Universiteit Leuven Campus Kortrijk, Universitaire Campus, B-8500 Kortrijk, Belgium

E-mail address: Karel.Dekimpe@kulak.ac.be

E-mail address: Wim.Malfait@kulak.ac.be 